\title{
Conditions for the cosmological viability of the most general scalar-tensor theories and their applications to extended Galileon dark energy models
}

\author{
Antonio De Felice ${ }^{1,2}$ and Shinji Tsujikawa ${ }^{3}$ \\ ${ }^{1}$ TPTP \& NEP, The Institute for Fundamental Study, \\ Naresuan University, Phitsanulok 65000, Thailand \\ ${ }^{2}$ Thailand Center of Excellence in Physics, Ministry of Education, Bangkok 10400, Thailand \\ ${ }^{3}$ Department of Physics, Faculty of Science, Tokyo University of Science, \\ 1-3, Kagurazaka, Shinjuku-ku, Tokyo 162-8601, Japan
}

(Dated: October 19, 2011)

\begin{abstract}
In the Horndeski's most general scalar-tensor theories with second-order field equations, we derive the conditions for the avoidance of ghosts and Laplacian instabilities associated with scalar, tensor, and vector perturbations in the presence of two perfect fluids on the flat Friedmann-LemaitreRobertson-Walker (FLRW) background. Our general results are useful for the construction of theoretically consistent models of dark energy. We apply our formulas to extended Galileon models in which a tracker solution with an equation of state smaller than -1 is present. We clarify the allowed parameter space in which the ghosts and Laplacian instabilities are absent and we numerically confirm that such models are indeed cosmologically viable.
\end{abstract}

\section{INTRODUCTION}

The problem of dark energy is one of the most serious problems faced by cosmologists and particle physicists. The cosmological constant is a simplest candidate, but it is plagued by the huge difference of energy scales between the theoretical and observed values [1]. Many alternative models to the cosmological constant have been proposed to approach the origin of dark energy [2].

Dark energy models can be broadly classified into two classes: (i) modified matter models, and (ii) modified gravity models. In the class (i) the modified matter source is introduced to drive the late-time cosmic acceleration. The representative model in this class is quintessence [3], in which the potential energy $V(\phi)$ of a slow-rolling scalar field $\phi$ is the source for dark energy. Another model is k-essence [4], in which a non-linear term with respect to the field kinetic energy $X=-(\partial \phi)^{2} / 2$ leads to the cosmic acceleration.

The representative models that belong to the class (ii) are those based on $f(R)$ theories [ [5, [], $f(R, \mathcal{G})$ theories $|7|$, Brans-Dicke theories [8], Dvali-Gabadazde-Porrati (DGP) braneworld [9], and Galileon gravity [10, 11] (see Ref. [12] for reviews). The Lagrangian in $f(R)$ theories is an arbitrary function of the Ricci scalar $R$, which corresponds to the particular class of Brans-Dicke theories (Brans-Dicke parameter $\omega_{\mathrm{BD}}=0[13]$ ). The $f(R, \mathcal{G})$ theories consist of a function of $R$ and the Gauss-Bonnet term $\mathcal{G}$, in which case there are two independent scalar degrees of freedom in a general background [14]. In Galileon gravity, the Lagrangian is constructed such that the field equations are invariant under the Galilean symmetry $\partial_{\mu} \phi \rightarrow \partial_{\mu} \phi+b_{\mu}$ in the limit of Minkowski spacetime [10]. One of those terms is given by $X \square \phi$, which appears in the DGP model as a result of the mixing of a brane-bending mode with a transverse graviton [15]. In the Galileon model there exists a stable late-time de Sitter solution where $\dot{\phi}=$ constant [16, 17]. Moreover the model can be consistent with local gravity constraints through the Vainshtein mechanism [18].

Recently Deffayet et al. [19] derived the action for the most general scalar-tensor theories having second-order field equations. This action is equivalent to that derived by Horndeski [20] in 1974 in the context of Lovelock gravity [21] (see also Ref. [22]). In fact the Horndeski's action is sufficiently general to accommodate most of the dark energy models proposed in literature. Moreover, the Gauss-Bonnet couplings $F(\phi) \mathcal{G}$ [23], the generalized Galileon term $G(\phi, X) \square \phi$ [24 27], the derivative couplings $G^{\mu \nu} \partial_{\mu} \phi \partial_{\nu} \phi$ with the Einstein tensor $G^{\mu \nu}$ [28] also belong to the class of the Horndeski's action [21].

For such general theories the full background and perturbation equations were recently derived in Ref. [29] in the presence of non-relativistic matter. In the context of inflation several authors computed the power spectra of scalar and tensor perturbations [21] as well as the non-linear parameter of primordial non-Gaussianities [30 32].

If we modify gravity from General Relativity we need to worry about the presence of ghosts as well as Laplacian instabilities. In the presence of two perfect fluids (radiation and non-relativistic matter) we shall derive conditions for the avoidance of ghosts and Laplacian instabilities associated with scalar, tensor, and vector perturbations in the Horndeski's theories. This is of particular importance for the construction of theoretically consistent modified gravitational models of dark energy. We apply our results to extended Galileon models in which a tracker solution is present. This tracker corresponds to the generalization of that found in Ref. [16] for the covariant Galileon. Unlike the covariant Galileon the equation of state of dark energy $w_{\mathrm{DE}}$ can take the values close to -1 during the evolution 
from the matter era to the accelerated epoch. This property should allow for the tracker to be compatible with observations.

This paper is organized as follows. In Sec. II the background equations of motion are derived on the flat FLRW spacetime in the presence of two perfect fluids. In Sec. III we find conditions for the absence of ghosts and Laplacian instabilities by deriving the second-order action for scalar, tensor, and vector perturbations. In Sec. IV we apply our general formulas to kinetically driven dark energy models, which cover the covariant Galileon as a specific case. Not only we identify a theoretically consistent parameter space but also we numerically integrate the field equations to confirm the analytic estimation. Sec. $\mathrm{V}$ is devoted to conclusions.

\section{THE BACKGROUND EQUATIONS OF MOTION}

In order to avoid the Ostrogradski instability [33] it is desirable to keep the equations of motion at second order in derivatives. The most general 4-dimensional scalar-tensor theories having second-order field equations are described by the Lagrangian [19]

$$
\mathcal{L}=\sum_{i=2}^{5} \mathcal{L}_{i}
$$

where

$$
\begin{aligned}
& \mathcal{L}_{2}=K(\phi, X), \\
& \mathcal{L}_{3}=-G_{3}(\phi, X) \square \phi, \\
& \mathcal{L}_{4}=G_{4}(\phi, X) R+G_{4, X}\left[(\square \phi)^{2}-\left(\nabla_{\mu} \nabla_{\nu} \phi\right)\left(\nabla^{\mu} \nabla^{\nu} \phi\right)\right], \\
& \mathcal{L}_{5}=G_{5}(\phi, X) G_{\mu \nu}\left(\nabla^{\mu} \nabla^{\nu} \phi\right)-\frac{1}{6} G_{5, X}\left[(\square \phi)^{3}-3(\square \phi)\left(\nabla_{\mu} \nabla_{\nu} \phi\right)\left(\nabla^{\mu} \nabla^{\nu} \phi\right)+2\left(\nabla^{\mu} \nabla_{\alpha} \phi\right)\left(\nabla^{\alpha} \nabla_{\beta} \phi\right)\left(\nabla^{\beta} \nabla_{\mu} \phi\right)\right] .
\end{aligned}
$$

Here $K$ and $G_{i}(i=3,4,5)$ are functions with respect to a scalar field $\phi$ and its kinetic energy $X=-\partial^{\mu} \phi \partial_{\mu} \phi / 2, R$ is the Ricci scalar, and $G_{\mu \nu}$ is the Einstein tensor. $G_{i, X}$ and $G_{i, \phi}(i=3,4,5)$ correspond to the partial derivatives of $G_{i}$ with respect to $X$ and $\phi$ respectively, i.e. $G_{i, X} \equiv \partial G_{i} / \partial X$ and $G_{i, \phi} \equiv \partial G_{i} / \partial \phi$. The above Lagrangian was first discovered by Horndeski in a different form [20], which is equivalent to the Lagrangian (10) [21].

In addition to the scalar field $\phi$ we take into account two barotropic perfect fluids, whose energy densities are $\rho_{A}$ and $\rho_{B}$. Then the total action is given by

$$
S=\int d^{4} x \sqrt{-g}\left(\mathcal{L}+\mathcal{L}_{A}+\mathcal{L}_{B}\right)
$$

where $g$ is the determinant of the metric $g_{\mu \nu}, \mathcal{L}_{A}$ and $\mathcal{L}_{B}$ are the Lagrangians of two perfect fluids respectively.

Let us consider a flat FLRW background with the metric $d s^{2}=-N^{2}(t) d t^{2}+a^{2}(t) d \boldsymbol{x}^{2}$, where $t$ is the cosmic time, $N(t)$ is the lapse function, and $a(t)$ is the scale factor. Varying the action (6) with respect to the $N(t)$ and $a(t)$ respectively, we obtain

$$
\begin{aligned}
& 2 X K_{, X}-K+6 X \dot{\phi} H G_{3, X}-2 X G_{3, \phi}-6 H^{2} G_{4}+24 H^{2} X\left(G_{4, X}+X G_{4, X X}\right)-12 H X \dot{\phi} G_{4, \phi X}-6 H \dot{\phi} G_{4, \phi} \\
& +2 H^{3} X \dot{\phi}\left(5 G_{5, X}+2 X G_{5, X X}\right)-6 H^{2} X\left(3 G_{5, \phi}+2 X G_{5, \phi X}\right)=-\rho_{A}-\rho_{B}, \\
& K-2 X\left(G_{3, \phi}+\ddot{\phi} G_{3, X}\right)+2\left(3 H^{2}+2 \dot{H}\right) G_{4}-12 H^{2} X G_{4, X}-4 H \dot{X} G_{4, X}-8 \dot{H} X G_{4, X}-8 H X \dot{X} G_{4, X X} \\
& +2(\ddot{\phi}+2 H \dot{\phi}) G_{4, \phi}+4 X G_{4, \phi \phi}+4 X(\ddot{\phi}-2 H \dot{\phi}) G_{4, \phi X}-2 X\left(2 H^{3} \dot{\phi}+2 H \dot{H} \dot{\phi}+3 H^{2} \ddot{\phi}\right) G_{5, X}-4 H^{2} X^{2} \ddot{\phi} G_{5, X X} \\
& +4 H X(\dot{X}-H X) G_{5, \phi X}+2\left[2(\dot{H} X+H \dot{X})+3 H^{2} X\right] G_{5, \phi}+4 H X \dot{\phi} G_{5, \phi \phi}=-p_{A}-p_{B},
\end{aligned}
$$

where a dot represents a derivative with respect to $t, H \equiv \dot{a} / a$ is the Hubble parameter, and $p_{A}, p_{B}$ are the pressures of the two perfect fluids. Varying the action (6) with respect to $\phi(t)$, it follows that

$$
\frac{1}{a^{3}} \frac{d}{d t}\left(a^{3} J\right)=P_{\phi}
$$

where

$$
\begin{aligned}
J \equiv & \dot{\phi} K_{, X}+6 H X G_{3, X}-2 \dot{\phi} G_{3, \phi}+6 H^{2} \dot{\phi}\left(G_{4, X}+2 X G_{4, X X}\right)-12 H X G_{4, \phi X} \\
& +2 H^{3} X\left(3 G_{5, X}+2 X G_{5, X X}\right)-6 H^{2} \dot{\phi}\left(G_{5, \phi}+X G_{5, \phi X}\right) \\
P_{\phi} \equiv & K_{, \phi}-2 X\left(G_{3, \phi \phi}+\ddot{\phi} G_{3, \phi X}\right)+6\left(2 H^{2}+\dot{H}\right) G_{4, \phi}+6 H(\dot{X}+2 H X) G_{4, \phi X} \\
& -6 H^{2} X G_{5, \phi \phi}+2 H^{3} X \dot{\phi} G_{5, \phi X} .
\end{aligned}
$$


The two perfect fluids obey the following continuity equations

$$
\begin{aligned}
& \dot{\rho}_{A}+3 H\left(\rho_{A}+p_{A}\right)=0, \\
& \dot{\rho}_{B}+3 H\left(\rho_{B}+p_{B}\right)=0 .
\end{aligned}
$$

Equations (7), (8), (9), (12), and (13) are not independent because of the Bianchi identities. The field equation (9) can be derived by using the other equations.

\section{CONDITIONS FOR THE AVOIDANCE OF GHOSTS AND LAPLACIAN INSTABILITIES IN THE PRESENCE OF TWO PERFECT FLUIDS}

In this section we study the stability of the flat FLRW background for the action (6) in the presence of two perfect fluids.

Let us first consider scalar metric perturbations $\Psi, \chi$, and $\Phi$ with the line element [34]

$$
d s^{2}=-(1+2 \Psi) d t^{2}+2 \partial_{i} \chi d t d x^{i}+(1+2 \Phi) d x^{2} .
$$

Here we have gauged away a scalar perturbation $E$ that appears in the form $E_{, i j}$ in front of the term $d \boldsymbol{x}^{2}$. This fixes the spatial part of the gauge-transformation vector $\xi^{\mu}$. In the following we choose the uniform-field gauge for which the field $\phi$ has no perturbations, that is, $\delta \phi=0$. This fixes the temporal part of the vector $\xi^{\mu}$.

The two perfect fluids can be described in terms of the following Lagrangian

$$
S_{\mathrm{pf}}=\int d^{4} x \sqrt{-g}\left[p_{A}\left(\mu_{A}, s_{A}\right)+p_{B}\left(\mu_{B}, s_{B}\right)\right]
$$

where $\mu_{i}$ and $s_{i}(i=A, B)$ correspond to the chemical potential and the entropy per particle respectively. We will employ the method introduced in Ref. [35] to study the perfect fluid from a Lagrangian point of view in order to extract the conditions for the absence of ghost and Laplace instabilities. In the following we will summarize and simplify the method given in Ref. [35] (see also Ref. [36]).

Since we are interested in those fluids with equations of state of the kind $p_{i}=w_{i} \rho_{i}(i=A, B)$, we consider $p_{i}$ as functions of $\mu_{i}$ alone. Here the chemical potential of each fluid is defined as $\mu_{i} n_{i}=\rho_{i}+p_{i}$, where $n_{i}=\partial p_{i} / \partial \mu_{i}$ is the number density of the fluid $i$. In fact, it is sufficient to give the equations of state $\mu_{i} \propto n_{i}^{w_{i}}$. We define the fluid 4 -velocity $u_{\alpha}$ associated with the chemical potential $\mu$, as $u_{\alpha}=\mu^{-1} \partial_{\alpha} \ell$, where $\ell$ is a scalar field. The normalization condition for the 4 -velocity allows us to write the 4 -velocity of the fluid $A$, as $\mu_{A}=\sqrt{-g^{\alpha \beta} \partial_{\alpha} \ell_{A} \partial_{\beta} \ell_{A}}$. After we perturb the field $\ell_{A}$ as $\ell_{A}(t)+\delta \ell_{A}$, we can expand the matter Lagrangian at second order and then perform the field redefinition $\delta \ell_{A}=-\mu_{A} v_{A}$, where $v_{A}$ is chosen to represent the independent scalar degree of freedom of the fluid $A$. Along the same lines, the independent scalar degree of freedom for the fluid $B$ corresponds to $v_{B}$. Since, at linear order, the scalar fluids do not contribute to the vector perturbations, it is sufficient to study their scalar contributions alone in order to derive the conditions for the avoidance of ghosts and Laplacian instabilities. We will discuss this issue in Appendix A.

We perturb the action $S=\int d^{4} x \sqrt{-g} \mathcal{L}+S_{\mathrm{pf}}$ up to the second order. After integrations by parts, the second-order action is given by

$$
\begin{aligned}
S^{(2)}=\int d^{4} x a^{3}[ & \left\{2 w_{1} \dot{\Phi}-w_{2} \Psi+\sum_{l} \rho_{l}\left(1+w_{l}\right) v_{l}\right\} \frac{\partial^{2} \chi}{a^{2}}+\left(\frac{1}{2} \sum_{l} \frac{\left(1+w_{l}\right) \rho_{l}}{w_{l}}+\frac{w_{3}}{3}\right) \Psi^{2}+\frac{w_{4}}{a^{2}}(\partial \Phi)^{2}-3 w_{1} \dot{\Phi}^{2} \\
& +\left\{3 w_{2} \dot{\Phi}-2 w_{1} \frac{\partial^{2} \Phi}{a^{2}}-\sum_{l} \frac{\rho_{l}\left(1+w_{l}\right)\left(\dot{v}_{l}-3 H w_{l} v_{l}\right)}{w_{l}}\right\} \Psi+\sum_{l} \frac{\rho_{l}\left(1+w_{l}\right)}{2 w_{l}}\left\{\dot{v}_{l}^{2}-\frac{w_{l}}{a^{2}}\left(\partial v_{l}\right)^{2}\right\} \\
& \left.+3 \Phi \sum_{l} \rho_{l}\left(1+w_{l}\right)\left(\dot{v}_{l}-3 H w_{l} v_{l}\right)+\frac{3}{2} \dot{H} \sum_{l}\left(1+w_{l}\right) \rho_{l} v_{l}^{2}\right]
\end{aligned}
$$


where $l=A, B$, and

$$
\begin{aligned}
w_{1} \equiv & 2\left(G_{4}-2 X G_{4, X}\right)-2 X\left(G_{5, X} \dot{\phi} H-G_{5, \phi}\right) \\
w_{2} \equiv & -2 G_{3, X} X \dot{\phi}+4 G_{4} H-16 X^{2} G_{4, X X} H+4\left(\dot{\phi} G_{4, \phi X}-4 H G_{4, X}\right) X+2 G_{4, \phi} \dot{\phi} \\
& +8 X^{2} H G_{5, \phi X}+2 H X\left(6 G_{5, \phi}-5 G_{5, X} \dot{\phi} H\right)-4 G_{5, X X} \dot{\phi} X^{2} H^{2}, \\
w_{3} \equiv & 3 X\left(K_{, X}+2 X K_{, X X}\right)+6 X\left(3 X \dot{\phi} H G_{3, X X}-G_{3, \phi X} X-G_{3, \phi}+6 H \dot{\phi} G_{3, X}\right) \\
& +18 H\left(4 H X^{3} G_{4, X X X}-H G_{4}-5 X \dot{\phi} G_{4, \phi X}-G_{4, \phi} \dot{\phi}+7 H G_{4, X} X+16 H X^{2} G_{4, X X}-2 X^{2} \dot{\phi} G_{4, \phi X X}\right) \\
& +6 H^{2} X\left(2 H \dot{\phi} G_{5, X X X} X^{2}-6 X^{2} G_{5, \phi X X}+13 X H \dot{\phi} G_{5, X X}-27 G_{5, \phi X} X+15 H \dot{\phi} G_{5, X}-18 G_{5, \phi}\right), \\
w_{4} \equiv & 2 G_{4}-2 X G_{5, \phi}-2 X G_{5, X} \ddot{\phi} .
\end{aligned}
$$

The equation of motion for $\chi$ gives rise to the following momentum constraint

$$
w_{2} \Psi=2 w_{1} \dot{\Phi}+\sum_{l=A, B} \rho_{l}\left(1+w_{l}\right) v_{l}
$$

which can be used to integrate out the field $\Psi$. After replacing $\Psi$ in Eq. (16), by using Eq. (21), we need to integrate the terms $\partial^{2} \Phi \dot{\Phi}$ and $\partial^{2} \Phi v_{l}$ by parts, so that the action (16) reduces to

$$
S^{(2)}=\int d^{4} x\left[\mathcal{A}_{i j} \dot{\mathcal{Q}}_{i} \dot{\mathcal{Q}}_{j}-\mathcal{C}_{i j}\left(\partial \mathcal{Q}_{i}\right)\left(\partial \mathcal{Q}_{j}\right)-\mathcal{B}_{i j} \mathcal{Q}_{i} \dot{\mathcal{Q}}_{j}-\mathcal{D}_{i j} \mathcal{Q}_{i} \mathcal{Q}_{j}\right]
$$

where $\mathcal{Q}_{i}=\left(\Phi, v_{A}, v_{B}\right)$, and $\mathcal{A}_{i j}, \mathcal{B}_{i j}, \mathcal{C}_{i j}, \mathcal{D}_{i j}$ are the components of the $3 \times 3$ matrices $\mathcal{A}, \mathcal{B}, \mathcal{C}, \mathcal{D}$.

Imposing the matrix $\mathcal{A}$ to be positive definite leads to the no-ghost conditions

$$
Q_{S} \equiv \frac{w_{1}\left(4 w_{1} w_{3}+9 w_{2}^{2}\right)}{3 w_{2}^{2}}>0
$$

and $w_{A}\left(1+w_{A}\right) \rho_{A}>0, w_{B}\left(1+w_{B}\right) \rho_{B}>0$. The latter two conditions are automatically satisfied for radiation $\left(w_{A}=1 / 3\right)$ and non-relativistic matter $\left(w_{B}=0^{+}\right)$. The speed of propagation $c_{s}$ for the fields can be found by solving the following discriminant equation

$$
\operatorname{det}\left(c_{s}^{2} \mathcal{A}-a^{2} \mathcal{C}\right)=0
$$

This has two trivial solutions $c_{s}^{2}=w_{A}$ and $c_{s}^{2}=w_{B}$, which are not negative for radiation and non-relativistic matter. In order to avoid the Laplacian instability associated with the remaining solution of Eq. (24) we require that

$$
c_{S}^{2} \equiv \frac{3\left(2 w_{1}^{2} w_{2} H-w_{2}^{2} w_{4}+4 w_{1} w_{2} \dot{w}_{1}-2 w_{1}^{2} \dot{w}_{2}\right)-6 w_{1}^{2}\left[\left(1+w_{A}\right) \rho_{A}+\left(1+w_{B}\right) \rho_{B}\right]}{w_{1}\left(4 w_{1} w_{3}+9 w_{2}^{2}\right)} \geq 0 .
$$

We also consider tensor perturbations $h_{i j}$ characterized by $\delta g_{i j}=a^{2}(t) h_{i j}$, where $h_{i j}$ is traceless $\left(h^{i}{ }_{i}=0\right)$ and divergence-free $\left(h^{i j}{ }_{j}=0\right)$. We decompose $h_{i j}$ into the two polarization modes, $h_{i j}=h_{\oplus} e_{i j}^{\oplus}+h_{\otimes} e_{i j}^{\otimes}$, where the matrices $e_{i j}^{\oplus}$ and $e_{i j}^{\otimes}$ are normalized to be $e_{i j}^{\lambda}(\boldsymbol{k}) e_{i j}^{\lambda}(-\boldsymbol{k})^{*}=2$, (where $\left.\lambda=\oplus, \otimes\right)$ and $e_{i j}^{\oplus}(\boldsymbol{k}) e_{i j}^{\otimes}(-\boldsymbol{k})^{*}=0$ in Fourier space. The second-order action for tensor perturbations can be written as

$$
S_{T}^{(2)}=\sum_{\lambda} \int d^{4} x a^{3} Q_{T}\left[\dot{h}_{\lambda}^{2}-\frac{c_{T}^{2}}{a^{2}}\left(\partial h_{\lambda}\right)^{2}\right]
$$

where the conditions for the avoidance of ghosts and Laplacian instabilities are given by

$$
\begin{aligned}
Q_{T} & \equiv \frac{w_{1}}{4}>0 \\
c_{T}^{2} & \equiv \frac{w_{4}}{w_{1}} \geq 0 .
\end{aligned}
$$

For the consistency of the theories given by the action (6) the conditions (23), (25), (27), and (28) need to be satisfied. Finally, as we will show in Appendix A, the vector modes do not add any conditions to those derived above. 


\section{APPLICATION TO EXTENDED GALILEON DARK ENERGY MODELS}

In this section we shall study the cosmology based on the extended Galileon models by taking into account the conditions for the avoidance of ghosts and Laplacian instabilities derived in Sec. III.

The covariant Galileon without the field potential [11] corresponds to

$$
K=-c_{2} X, \quad G_{3}=\frac{c_{3}}{M^{3}} X, \quad G_{4}=\frac{1}{2} M_{\mathrm{pl}}^{2}-\frac{c_{4}}{M^{6}} X^{2}, \quad G_{5}=\frac{3 c_{5}}{M^{9}} X^{2}
$$

where $c_{i}(i=2,3,4,5)$ are dimensionless constants, $M_{\mathrm{pl}}$ is the reduced Planck mass, and $M$ is a constant having the dimension of mass. In this case it is known that there exists a cosmological tracker solution characterized by the condition $H \dot{\phi}=$ constant [16]. Along the tracker the dark energy equation of state evolves as $w_{\mathrm{DE}}=-7 / 3$ (radiation era $\rightarrow w_{\mathrm{DE}}=-2$ (matter era) $\rightarrow w_{\mathrm{DE}}=-1$ (de Sitter era). However, the tracker solution for the covariant Galileon is not favored by the joint data analysis of Supernovae Ia (SNIa), Cosmic Microwave Background (CMB), and Baryon Acoustic Oscillations (BAO) [37]. This comes from the unusual evolution of $w_{\mathrm{DE}}$ during the matter era away from -1 . Only the solutions that approach the tracker at late times can be allowed observationally.

Let us consider the generalization of the covariant Galileon to find tracker solutions with different $w_{\mathrm{DE}}$. We take the following functions

$$
K=-c_{2} M_{2}^{4\left(1-p_{2}\right)} X^{p_{2}}, \quad G_{3}=c_{3} M_{3}^{1-4 p_{3}} X^{p_{3}}, \quad G_{4}=\frac{1}{2} M_{\mathrm{pl}}^{2}-c_{4} M_{4}^{2-4 p_{4}} X^{p_{4}}, \quad G_{5}=3 c_{5} M_{5}^{-\left(1+4 p_{5}\right)} X^{p_{5}}
$$

where $c_{i}$ and $p_{i}(i=2,3,4,5)$ are dimensionless constants, and $M_{i}(i=2,3,4,5)$ are constants having dimensions of mass $^{1}$. For two perfect fluids we consider radiation (energy density $\rho_{A}=\rho_{r}$, equation of state $w_{A}=1 / 3$ ) and non-relativistic matter (energy density $\rho_{B}=\rho_{m}$, equation of state $w_{B}=0$ ). For the choice (30) the field equations (7) and (8) can be written as

$$
\begin{aligned}
& 3 H^{2} M_{\mathrm{pl}}^{2}=\rho_{\mathrm{DE}}+\rho_{m}+\rho_{r}, \\
& \left(3 H^{2}+2 \dot{H}\right) M_{\mathrm{pl}}^{2}=-p_{\mathrm{DE}}-\rho_{r} / 3,
\end{aligned}
$$

where

$$
\begin{aligned}
\rho_{\mathrm{DE}} \equiv & 2 X K_{, X}-K+6 H \dot{\phi} X G_{3, X}-6 H^{2} \tilde{G}_{4}+24 H^{2} X\left(G_{4, X}+X G_{4, X X}\right)+2 H^{3} \dot{\phi} X\left(5 G_{5, X}+2 X G_{5, X X}\right), \\
p_{\mathrm{DE}} \equiv & K-2 \ddot{\phi} X G_{3, X}+2\left(3 H^{2}+2 \dot{H}\right) \tilde{G}_{4}-12 H^{2} X G_{4, X}-4 H \dot{X} G_{4, X}-8 \dot{H} X G_{4, X}-8 H X \dot{X} G_{4, X X} \\
& -2 X\left(2 H^{3} \dot{\phi}+2 H \dot{H} \dot{\phi}+3 H^{2} \ddot{\phi}\right) G_{5, X}-4 H^{2} \ddot{\phi} X^{2} G_{5, X X}
\end{aligned}
$$

and $\tilde{G}_{4} \equiv G_{4}-M_{\mathrm{pl}}^{2} / 2=-c_{4} M_{4}^{2-4 p_{4}} X^{p_{4}}$

For the covariant Galileon $\left(p_{2}=p_{3}=1, p_{4}=p_{5}=2\right)$ there is a tracker solution with $H \dot{\phi}=$ constant, in which case all the terms in Eq. (33) are proportional to $\dot{\phi}^{2}$. We search for tracker solutions characterized by the condition

$$
H \dot{\phi}^{2 q}=\text { constant }
$$

where the power $q$ is a real constant. If we choose the following powers, all the terms in Eq. (33) are proportional to $\dot{\phi}^{2 p}$ :

$$
p_{2}=p, \quad p_{3}=p+(2 q-1) / 2, \quad p_{4}=p+2 q, \quad p_{5}=p+(6 q-1) / 2 .
$$

Note that the covariant Galileon corresponds to $p=1$ and $q=1 / 2$.

Let us study whether the tracker really exists or not for the powers given by Eq. (36). Before doing so we first discuss conditions for the existence of a de Sitter solution characterized by $\dot{\phi}=$ constant and $H=$ constant. We introduce the following variable

$$
x \equiv \frac{\dot{\phi}}{H M_{\mathrm{pl}}}
$$

\footnotetext{
${ }^{1}$ Kimura and Yamamoto [26] studied the model with $p_{2}=1, p_{3} \neq 0, c_{4}=0, c_{5}=0$, which recovers the Dvali-Turner model $\left.\underline{38}\right]$.
} 
For the simplification of the background equations it is convenient to define

$$
\begin{aligned}
& M_{2} \equiv\left(H_{\mathrm{dS}} M_{\mathrm{pl}}\right)^{1 / 2}, \quad M_{3} \equiv\left(\frac{M_{\mathrm{pl}}^{1-2 p_{3}}}{H_{\mathrm{dS}}^{2 p_{3}}}\right)^{1 /\left(1-4 p_{3}\right)}, \\
& M_{4} \equiv\left(\frac{M_{\mathrm{pl}}^{2-2 p_{4}}}{H_{\mathrm{dS}}{ }^{2 p_{4}}}\right)^{1 /\left(2-4 p_{4}\right)}, \quad M_{5} \equiv\left(\frac{H_{\mathrm{dS}}{ }^{2+2 p_{5}}}{M_{\mathrm{pl}}{ }^{1-2 p_{5}}}\right)^{1 /\left(1+4 p_{5}\right)},
\end{aligned}
$$

where $H_{\mathrm{dS}}$ is the Hubble parameter at the de Sitter solution. For the covariant Galileon one has $M_{3}=M_{4}=M_{5}=$ $\left(H_{\mathrm{dS}}^{2} M_{\mathrm{pl}}\right)^{1 / 3}$. From Eqs. (31) and (32) we find that the de Sitter fixed point where $\ddot{\phi}=0$ and $\dot{H}=0$ is present under the following conditions

$$
\begin{aligned}
& c_{2}=\frac{3}{2}\left(\frac{2}{x_{\mathrm{dS}}^{2}}\right)^{p}(3 \alpha-4 \beta+2), \\
& c_{3}=\frac{\sqrt{2}}{2 p+q-1}\left(\frac{2}{x_{\mathrm{dS}}^{2}}\right)^{p+q}[3(p+q)(\alpha-\beta)+p],
\end{aligned}
$$

where $x_{\mathrm{dS}}$ is the value of $x$ at the de Sitter solution, and

$$
\alpha \equiv \frac{4\left(2 p_{4}-1\right)}{3}\left(\frac{x_{\mathrm{dS}}^{2}}{2}\right)^{p_{4}} c_{4}, \quad \beta \equiv 2 \sqrt{2} p_{5}\left(\frac{x_{\mathrm{dS}}^{2}}{2}\right)^{p_{5}+1 / 2} c_{5} .
$$

In order to discuss the cosmological dynamics it is convenient to introduce the following variables

$$
r_{1} \equiv\left(\frac{x_{\mathrm{dS}}}{x}\right)^{2 q}\left(\frac{H_{\mathrm{dS}}}{H}\right)^{1+2 q}, \quad r_{2} \equiv\left[\left(\frac{x}{x_{\mathrm{dS}}}\right)^{2} \frac{1}{r_{1}^{3}}\right]^{\frac{p+2 q}{1+2 q}}, \quad \Omega_{r} \equiv \frac{\rho_{r}}{3 H^{2} M_{\mathrm{pl}}^{2}} .
$$

The solution (35), in terms of the variable $r_{1}$, is given by $r_{1}=1$. The de Sitter fixed point corresponds to $\left(r_{1}, r_{2}, \Omega_{r}\right)=$ $(1,1,0)$. We will only consider cosmological dynamics for which $r_{1}$ and $r_{2}$ are both positive at all times: otherwise the inverse relations of (42) may be ill-defined. On the other hand, we will see later on that a viable cosmology cannot allow a change of sign for the variable $r_{2}$. Defining the dark energy density parameter $\Omega_{\mathrm{DE}} \equiv \rho_{\mathrm{DE}} /\left(3 H^{2} M_{\mathrm{pl}}^{2}\right)$, it follows that

$$
\Omega_{\mathrm{DE}}=\frac{r_{1}^{\frac{p-1}{2 q+1}} r_{2}}{2}\left[r_{1}\left\{r_{1}\left[12(\alpha-\beta)(p+q)+4 p-r_{1}(2 p-1)(3 \alpha-4 \beta+2)\right]-3 \alpha(2 p+4 q+1)\right\}+4 \beta(p+3 q+1)\right] .
$$

The Friedmann equation gives the relation $\Omega_{m} \equiv \rho_{m} /\left(3 H^{2} M_{\mathrm{pl}}^{2}\right)=1-\Omega_{r}-\Omega_{\mathrm{DE}}$. For the initial conditions where $r_{1}$ is positive but small $\left(0<r_{1} \ll 1\right)$, we require that

$$
\frac{p-1}{2 q+1} \geq 0
$$

in order to have $\Omega_{\mathrm{DE}} \rightarrow 0$. In the following we will replace $\Omega_{m}$ with $1-\Omega_{r}-\Omega_{\mathrm{DE}}$.

We can obtain the differential equations for $r_{1}, r_{2}$, and $\Omega_{r}$ after deriving $\ddot{\phi}$ and $\dot{H}$ from Eqs. (8) and (9), e.g., $r_{1}^{\prime} / r_{1}=-2 q \ddot{\phi} /(H \dot{\phi})-\dot{H} / H^{2}$, where a prime represents a derivative with respect to $N=\ln a$. The r.h.s. of these differential equations can be expressed in terms of $r_{1}, r_{2}$, and $\Omega_{r}$ together with the coefficients $\alpha, \beta, p$, and $q$.

The equation for $r_{1}$ is given by

$$
\begin{aligned}
r_{1}^{\prime}= & {\left[r_{1}\left(r_{1}-1\right)\left\{\beta\left(2 p\left(r_{1}-1\right)\left(2 r_{1}+1\right)-6 q\left(r_{1}+1\right)\right)+r_{1}\left(3 \alpha\left(p-p r_{1}+2 q\right)-2 p r_{1}\right)\right\}\right.} \\
& \times\left\{2\left(\left(3+\Omega_{r}\right)(2 p-1)+12 q\right) r_{1}^{(1-p) /(1+2 q)}+3\left(4 \beta(1+p+3 q)-3 \alpha(1+2 p+4 q) r_{1}\right.\right. \\
& \left.\left.\left.+4((1+3 \alpha-3 \beta) p+3(\alpha-\beta) q) r_{1}^{2}+(-2-3 \alpha+4 \beta)(2 p-1) r_{1}^{3}\right) r_{2}\right\}\right] / \Delta
\end{aligned}
$$

where

$$
\begin{aligned}
\Delta \equiv & 2\left[-2 r_{1}^{(2+2 q-p) /(1+2 q)}\left(3 \alpha(p+2 q)(2 p+4 q-1)-(2 p+2 q-1)((2+6 \alpha) p+6 \alpha q) r_{1}+(2+3 \alpha) p(2 p-1) r_{1}^{2}\right)\right. \\
& +4 \beta r_{1}^{(1-p) /(1+2 q)}\left(2 p^{2}-p+18 q^{2}+12 p q-3 q-3(2 p+2 q-1)(p+q) r_{1}^{2}+2 p(2 p-1) r_{1}^{3}\right) \\
& +r_{1}^{2}\left\{9 \alpha^{2}\left(2 q\left(2 q r_{1}-2 q-1\right)+\left(2 p^{2}+p+8 p q\right)\left(r_{1}-1\right)\right)\left(r_{1}-1\right)+4 p^{2} r_{1}^{2}+6 \alpha p r_{1}\left((2 p+4 q+1) r_{1}-2 p-6 q-1\right)\right\} r_{2} \\
& +3 \beta^{2}\left(r_{1}-1\right)\left\{4 p\left(r_{1}-1\right)\left(1+2 r_{1}+6 q\left(r_{1}+1\right)^{2}\right)+4 p^{2}\left(r_{1}-1\right)\left(1+r_{1}\left(2+3 r_{1}\right)\right)+6 q\left(r_{1}+1\right)\left(2 q\left(r_{1}^{2}-3\right)-2\right)\right\} r_{2} \\
& -3 \beta r_{1}^{2}\left\{4 p r_{1}\left(\left(r_{1}-1\right)\left(2 p r_{1}+1\right)+2 q\left(r_{1}^{2}-2\right)\right)+\alpha\left(r_{1}-1\right)\left(8 p^{2}\left(r_{1}-1\right)\left(2 r_{1}+1\right)+6 q\left(4 q r_{1}^{2}-(1+2 q) r_{1}-3-10 q\right)\right.\right. \\
& \left.\left.\left.+4 p\left(r_{1}-1\right)\left(12 q\left(r_{1}+1\right)+r_{1}+2\right)\right)\right\} r_{2}\right] .
\end{aligned}
$$


In addition to Eq. (45) we write the remaining equations of motion for the variables $r_{2}$ and $\Omega_{r}$. We find that the second Einstein equation is equivalent to

$$
\begin{aligned}
& \frac{r_{1}^{\prime}}{r_{1}}\left\{4(3 q+1)(p+2 q)-2 r_{2}(p+2 q) r_{1}^{\frac{p-1}{2 q+1}}\left\{3 \alpha r_{1}(p-q-1)-3 \beta(p-3 q-2)-r_{1}^{2}[3 \alpha p-3 \beta(p+q)+p+3 \alpha q]\right\}\right\} \\
& +\frac{2 r_{2}^{\prime}}{r_{2}}(2 q+1)\left\{r_{1}^{\frac{p-1}{2 q+1}} r_{2}\left\{3 \beta(p+q)+r_{1}^{2}[3 \alpha p-3 \beta(p+q)+p+3 \alpha q]-3 \alpha r_{1}(p+q)\right\}+2 q\right\} \\
& \quad-3(2 q+1)(p+2 q)\left\{r_{1}^{\frac{p-1}{2 q+1}} r_{2}\left[r_{1}^{3}(4 \beta-3 \alpha-2)+3 \alpha r_{1}-4 \beta\right]+2\right\}-2(2 q+1)(p+2 q) \Omega_{r}=0
\end{aligned}
$$

whereas the equation of continuity for the radiation fluid leads to

$$
(2 q+1)(p+2 q) \frac{\Omega_{r}^{\prime}}{\Omega_{r}}-2(3 q+1)(p+2 q) \frac{r_{1}^{\prime}}{r_{1}}-2 q(2 q+1) \frac{r_{2}^{\prime}}{r_{2}}+4(2 q+1)(p+2 q)=0 .
$$

These two equations, combined with Eq. (45), completely determine the dynamics of the system.

\section{A. Tracker}

From Eq. (45) we find that there is a fixed point characterized by

$$
r_{1}=1
$$

which corresponds to the tracker solution with $H \dot{\phi}^{2 q}=$ constant. Considering the homogenous perturbations $\delta r_{1}$ along the solution $r_{1}=1$, it follows that

$$
\delta r_{1}^{\prime}=-\frac{6(p+2 q)-3+(2 p-1) \Omega_{r}+3 r_{2}}{2\left(p r_{2}+2 q\right)} \delta r_{1}
$$

For $p r_{2}+2 q>0$ the tracker is stable along the $r_{1}$ direction provided that

$$
6(p+2 q)+(2 p-1) \Omega_{r}>3\left(1-r_{2}\right) .
$$

Along the tracker the dark energy density parameter is simply given by

$$
\Omega_{\mathrm{DE}}=r_{2}
$$

For $0 \leq \Omega_{\mathrm{DE}} \leq 1$ the r.h.s. of Eq. (51) is within the range $0 \leq 3\left(1-r_{2}\right) \leq 3$. As long as the condition (51) is satisfied, which includes the case of the covariant Galileon, the solutions stay at the tracker. With the increase of $r_{2}$ the solutions finally reach the de Sitter fixed point characterized by $r_{1}=1$ and $r_{2}=1$.

Along the tracker the equations for $r_{2}$ and $\Omega_{r}$ are given by

$$
\begin{aligned}
r_{2}^{\prime} & =\frac{(p+2 q)\left(\Omega_{r}+3-3 r_{2}\right)}{p r_{2}+2 q} r_{2}, \\
\Omega_{r}^{\prime} & =\frac{2 q\left(\Omega_{r}-1-3 r_{2}\right)-4 p r_{2}}{p r_{2}+2 q} \Omega_{r} .
\end{aligned}
$$

Combining these equations, it follows that

$$
\frac{r_{2}^{\prime}}{r_{2}}-(1+s) \frac{\Omega_{r}^{\prime}}{\Omega_{r}}=4(1+s), \quad \text { where } \quad s \equiv \frac{p}{2 q} .
$$

Integration of this equation leads to

$$
r_{2}=c_{1} a^{4(1+s)} \Omega_{r}^{1+s}
$$

where $c_{1}$ is a constant. Since $\Omega_{r} \propto a^{-4} H^{-2}$, the evolution of the variable $r_{2}$ is $r_{2} \propto H^{-2(1+s)}$. Since we want $r_{2}$ to be subdominant at early times and to grow on the tracker solution, we further impose

$$
1+s=1+\frac{p}{2 q}>0
$$


Substituting Eq. (56) into (54), we obtain the integrated solution

$$
c_{1} a^{4(1+s)} \Omega_{r}^{1+s}=1-\Omega_{r}\left(1-c_{2} a\right),
$$

where $c_{2}$ is a constant. Therefore, the dynamics on the tracker depends only on the free parameter $s=p /(2 q)$ and the two initial conditions $c_{1,2}$. For a particular choice of $s$ such as $s=1$ (which corresponds to the covariant Galileon), it is possible to derive the explicit solution for $\Omega_{r}$ in terms of $a$ [17, 37]. For general $s$, however, we cannot necessarily find an explicit expression for $\Omega_{r}$. The density parameter of dark energy along the tracker is given by $\Omega_{\mathrm{DE}}=r_{2}=c_{1} a^{4(1+s)} \Omega_{r}{ }^{1+s}$. Writing the density parameters of dark energy and radiation today $(a=1)$ as $\Omega_{\mathrm{DE}}^{(0)}$ and $\Omega_{r}^{(0)}$ respectively and using Eqs. (52), (56), and (58), the coefficients $c_{1}$ and $c_{2}$ are found to be

$$
c_{1}=\frac{1-\Omega_{m}^{(0)}-\Omega_{r}^{(0)}}{\left(\Omega_{r}^{(0)}\right)^{1+s}}, \quad c_{2}=-\frac{\Omega_{m}^{(0)}}{\Omega_{r}^{(0)}},
$$

where $\Omega_{m}^{(0)}=1-\Omega_{\mathrm{DE}}^{(0)}-\Omega_{r}^{(0)}$ is the density parameter of non-relativistic matter today.

The dark energy equation of state $w_{\mathrm{DE}} \equiv p_{\mathrm{DE}} / \rho_{\mathrm{DE}}$ and the effective total equation of state $w_{\mathrm{eff}} \equiv-1-2 \dot{H} /\left(3 H^{2}\right)$ along the tracker are

$$
w_{\mathrm{DE}}=-\frac{3+s\left(3+\Omega_{r}\right)}{3\left(s r_{2}+1\right)}, \quad w_{\mathrm{eff}}=-\frac{3 r_{2}(s+1)-\Omega_{r}}{3\left(s r_{2}+1\right)} .
$$

In the early cosmological epoch in which the condition $\Omega_{\mathrm{DE}}=r_{2} \ll 1$ is satisfied, one has $w_{\mathrm{DE}} \simeq-1-s\left(1+\Omega_{r} / 3\right)$ and $w_{\text {eff }} \simeq \Omega_{r} / 3$, respectively. Hence the evolution of $w_{\mathrm{DE}}$ during the radiation and matter eras is given by $w_{\mathrm{DE}}=-1-4 s / 3$ and $w_{\mathrm{DE}}=-1-s$, respectively. At the de Sitter fixed point $\left(r_{2}=1\right.$ and $\left.\Omega_{r}=0\right)$ it follows that $w_{\mathrm{DE}}=w_{\mathrm{eff}}=-1$. The tracker solution for the covariant Galileon $(s=1)$ is incompatible with observations because $w_{\mathrm{DE}}$ is away from -1 during the matter and radiation eras [37]. For the compatibility with observations we require that

$$
s=\frac{p}{2 q}<1
$$

In the regime $r_{2} \ll 1$ the conditions for the avoidance of ghosts and Laplacian instabilities are given by

$$
\begin{aligned}
\frac{Q_{S}}{M_{\mathrm{pl}}^{2} \simeq} & 6 q[p-3(\alpha-2 \beta) q] r_{2}>0 \\
c_{S}^{2} \simeq & \left\{4 p^{3}\left(\Omega_{r}+3\right)-2 p^{2}\left\{\left(\Omega_{r}+3\right)(6 \beta-3 \alpha+2)-2 q\left[3 \Omega_{r}+11-3(\alpha-2 \beta)\left(\Omega_{r}+3\right)\right]\right\}\right. \\
& -3\left\{\beta\left(\Omega_{r}+3\right)+8 q^{3}\left(\Omega_{r}+5\right)(\alpha-2 \beta)-2 q^{2}\left(7 \Omega_{r}+27\right)(\alpha-2 \beta)+q\left[3 \alpha\left(\Omega_{r}+3\right)-2 \beta\left(5 \Omega_{r}+17\right)\right]\right\} \\
& -p\left\{\left(\Omega_{r}+3\right)(3 \alpha-12 \beta-1)+4 q^{2}\left[(\alpha-2 \beta)\left(9 \Omega_{r}+33\right)-2\left(\Omega_{r}+5\right)\right]\right. \\
& \left.\left.+q\left[12(2 \beta-\alpha)\left(3 \Omega_{r}+10\right)+6 \Omega_{r}+22\right]\right\}\right\} /\left[24 q^{2}(2 p+4 q-1)\{p-3(\alpha-2 \beta) q\}\right] \geq 0 \\
\frac{Q_{T} \simeq}{M_{\mathrm{pl}}^{2} \simeq} & \frac{1}{8}\left[2+3(\alpha-2 \beta) r_{2}\right]>0, \\
c_{T}^{2} \simeq & 1-\frac{3\left\{2[2(\alpha-2 \beta) q+3 \beta] p+8(\alpha-2 \beta) q^{2}+\beta(16 q-3)+\beta(2 p+4 q-1) \Omega_{r}\right\}}{4 q(2 p+4 q-1)} r_{2} \geq 0
\end{aligned}
$$

These results reproduce those derived in Refs. [16, 17] for the covariant Galileon $(p=1, q=1 / 2)$. Let us consider the case in which the parameters $\alpha, \beta, p, q$ are not very different from the order of unity. Since $r_{2} \ll 1$ the conditions (64) and (65) are automatically satisfied. We see here that $r_{2}$ cannot change its sign, as this implies the violation of the condition $Q_{S}>0$. Since we only consider the case $r_{2}>0$ at the initial stage, the scalar ghost can be avoided for

$$
q[p-3(\alpha-2 \beta) q]>0 .
$$

For $p$ and $q$ satisfying $q(2 p+4 q-1)>0$, the Laplacian instability of the scalar perturbation is absent as long as the numerator in Eq. (63) is positive. For the covariant Galileon this corresponds to the condition $8+10 \alpha-9 \beta+\Omega_{r}(2+$ $3 \alpha-3 \beta) \geq 0[16,17]$.

\section{B. de Sitter solutions $\left(r_{1}=r_{2}=1\right)$}

We study the stability of the late-time de Sitter solution characterized by $r_{1}=r_{2}=1$. At the de Sitter fixed point the system is effectively described by one scalar degree of freedom $\Phi$, with the second-order action $S^{(2)}=$ 
$\int d^{4} x a^{3} Q_{S}\left[\dot{\Phi}^{2}-\left(c_{S}^{2} / a^{2}\right)(\nabla \Phi)^{2}\right]$. For homogenous perturbations (comoving wavenumber $k=0$ ) the scalar perturbation obeys the equation of motion $\frac{d}{d t}\left(a^{3} Q_{S} \dot{\Phi}\right)=0$, whose solution is

$$
\Phi(t)=c_{1}+c_{2} \int^{t} \frac{1}{a^{3} Q_{S}} d \tilde{t}
$$

where $c_{1}$ and $c_{2}$ are constants. Now we are considering the de Sitter solution where $\dot{\phi}$ is constant, in which case $Q_{S}$ does not vary in time. Since $a \propto e^{H_{\mathrm{ds}} t}$, the second term in Eq. (67) decays in proportion to $e^{-3 H_{\mathrm{dS}} t}$. Hence the de Sitter solution in our theory is classically stable against homogeneous perturbations.

The conditions for the avoidance of ghosts and Laplacian instabilities (against inhomogeneous perturbations) at $r_{1}=r_{2}=1$ are given by

$$
\begin{aligned}
\frac{Q_{S}}{M_{\mathrm{pl}}^{2}}= & \frac{6(p+2 q)(3 \alpha-6 \beta+2)[p-3(\alpha-2 \beta) q]}{[2 p-6(\alpha-2 \beta) q-3 \alpha+6 \beta-2]^{2}}>0 \\
c_{S}^{2}= & \left\{6 \beta+4 p^{2}+p\left[9(\alpha-2 \beta)^{2}+3 \alpha-12 \beta+4 q(6 \beta-3 \alpha+2)-2\right]+3(\alpha-2 \beta)[3 \beta+q(9 \alpha-12 \beta-8 q+6)]\right\} \\
& \times \frac{3(2 \beta-\alpha)(2 q+1)+2 p-2}{6(6 \beta-3 \alpha-2)(p+2 q)(2 p+4 q-1)(p-3 \alpha q+6 \beta q)} \geq 0 \\
\frac{Q_{T}}{M_{\mathrm{pl}}^{2}=} & \frac{1}{8}(3 \alpha-6 \beta+2)>0 \\
c_{T}^{2}= & \frac{2(2 p+4 q-1)-3 \alpha}{(2 p+4 q-1)(3 \alpha-6 \beta+2)} \geq 0
\end{aligned}
$$

\section{The solutions in the regime $r_{1} \ll 1$ and $r_{2} \ll 1$}

Equation (45) shows that there is another fixed point characterized by

$$
r_{1}=0 .
$$

Let us study the behavior of the solutions in the regime $r_{1} \ll 1$ and $r_{2} \ll 1$. In doing so we consider the parameter space with $p \geq 1$ and $q>0$. Then the equations for $r_{1}, r_{2}$, and $\Omega_{r}$ are approximately given by

$$
\begin{aligned}
r_{1}^{\prime} & \simeq \frac{\left(3+\Omega_{r}\right)(2 p-1)+12 q}{2(2 p+6 q-1)} r_{1}, \\
r_{2}^{\prime} & \simeq \frac{(p+2 q)\left[9-6 p+\Omega_{r}(7-2 p+12 q)\right]}{2(2 q+1)(2 p+6 q-1)} r_{2}, \\
\Omega_{r}^{\prime} & \simeq \Omega_{r}\left(\Omega_{r}-1\right) .
\end{aligned}
$$

From Eq. (75) there are two fixed points: $\Omega_{r}=1$ and $\Omega_{r}=0$. During the radiation era $\left(\Omega_{r}=1\right)$ integration of Eqs. (73) and (74) gives

$$
r_{1} \simeq a^{\frac{4 p+6 q-2}{2 p+6 q-1}}, \quad r_{2} \simeq a^{\frac{(p+2 q)(8-4 p+6 q)}{(2 q+1)(2 p+6 q-1)}},
$$

whereas during the matter era $\left(\Omega_{r}=0\right)$ one has

$$
r_{1} \simeq a^{\frac{3(2 p+4 q-1)}{2(2 p+6 q-1)}}, \quad r_{2} \simeq a^{\frac{3(p+2 q)(3-2 p)}{2(2 q+1)(2 p+6 q-1)}} .
$$

The variable $r_{1}$ increases for $p \geq 1$ and $q>0$, which is followed by the approach to the tracker solution. Whether the variable $r_{2}$ grows or not depends on the values of $p$ and $q$. If $p>3 / 2$ and $q>0$, for example, $r_{2}$ decreases in the matter era. However the dynamics will in general change as the solutions approach the tracker, $r_{1} \rightarrow 1$.

The dark energy equation of state $w_{\mathrm{DE}}$ and the total effective equation of state $w_{\text {eff }}$ in the regime $r_{1} \ll 1$ and $r_{2} \ll 1$ are approximately given by

$$
w_{\mathrm{DE}} \simeq \frac{1+\Omega_{r}}{2(1-2 p-6 q)}, \quad w_{\mathrm{eff}} \simeq \frac{1}{3} \Omega_{r} .
$$

If $p=1$ and $q=1 / 2$, we have $w_{\mathrm{DE}}=-\left(1+\Omega_{r}\right) / 8[16$, 17]. 
For $(p-1) /(2 q+1)>0$ the ghosts and Laplacian instabilities can be avoided for

$$
\begin{aligned}
\frac{Q_{S}}{M_{\mathrm{pl}}^{2}} & \simeq 3(p+3 q)(2 p+6 q-1) \beta r_{1}^{(p-1) /(2 q+1)} r_{2}>0, \\
c_{S}^{2} & \simeq \frac{p+3 q-2}{2(p+3 q)(2 p+6 q-1)}\left(1+\Omega_{r}\right) \geq 0, \\
\frac{Q_{T}}{M_{\mathrm{pl}}^{2}} & \simeq \frac{1}{4}\left[1-3 \beta r_{2} r_{1}^{(p-1) /(2 q+1)}\right]>0, \\
c_{T}^{2} & \simeq 1+\frac{3\left(4 p+12 q-5-3 \Omega_{r}\right)}{4 p+12 q-2} \beta r_{1}^{(p-1) /(2 q+1)} r_{2} \geq 0 .
\end{aligned}
$$

In the regime $r_{1} \ll 1$ and $r_{2} \ll 1$ the conditions (81) and (82) are automatically satisfied.

\section{Other fixed points}

From Eq. (45) we see that in general there are other two more complicated fixed points, $r_{1}=r_{a, b}$, those which satisfy the equation

$$
p(3 \alpha-4 \beta+2) r_{j}^{2}+[2 \beta(p+3 q)-3 \alpha(p+2 q)] r_{j}+2 \beta(p+3 q)=0,
$$

with $j=a, b$. Whether or not these fixed points are viable or not depends on their stability and the chosen parameters of the model.

Then we will consider only those models for which either there are no real solutions to Eq. (83), or, if they exist, they are placed outside the range of interest corresponding to the interval $0<r_{1} \leq 1$. Therefore we need to fulfill the following condition

$$
(\bar{\Delta}<0) \vee\left[\bar{\Delta} \geq 0 \wedge\left(r_{a}<0 \vee r_{a}>1\right) \wedge\left(r_{b}<0 \vee r_{b}>1\right)\right]
$$

where

$$
\begin{aligned}
r_{a, b} & =\frac{3 \alpha(p+2 q)-2 \beta(p+3 q) \pm \sqrt{\bar{\Delta}}}{2 p(3 \alpha-4 \beta+2)}, \\
\bar{\Delta} & =[2 \beta(p+3 q)-3 \alpha(p+2 q)]^{2}-8 \beta p(3 \alpha-4 \beta+2)(p+3 q) .
\end{aligned}
$$

\section{E. Theoretically viable parameter space}

Let us discuss the relevant constraints we have found so far in order to restrict the allowed parameter space. Therefore we have

$$
\begin{array}{rlrl}
\frac{p-1}{1+2 q} & \geq 0, & & \text { in order to have } \Omega_{\mathrm{DE}} \text { Subdominant et early times, } \\
1+\frac{p}{2 q} & >0, & & \text { in order to have a growing } \Omega_{\mathrm{DE}} \text { along the tracker }, \\
\frac{p}{2 q}<1, & & \text { in order to have the tracker consistent with data, } \\
\frac{p+3 q-2}{(p+3 q)(2 p+6 q-1)} & \geq 0, & & \text { in order to have }\left.c_{S}^{2}\right|_{r_{1} \ll 1, r_{2} \ll 1} \geq 0, \\
(p+3 q)(2 p+6 q-1) \beta & >0, & & \text { in order to have }\left.Q_{S}\right|_{r_{1} \ll 1, r_{2} \ll 1}>0, \\
3 \alpha-6 \beta+2 & >0, & & \text { in order to have }\left.Q_{T}\right|_{\text {de Sitter }}>0, \\
(p+2 q)[p-3(\alpha-2 \beta) q] & >0, & & \text { in order to have }\left.Q_{S}\right|_{\text {de Sitter }}>0, \\
q[p-3(\alpha-2 \beta) q] & >0, & & \text { in order to have }\left.Q_{S}\right|_{r_{1}=1, r_{2} \ll 1}>0, \\
\left.c_{S}^{2}\right|_{\text {de Sitter }} & \geq 0, & & \left.c_{T}^{2}\right|_{\text {de Sitter }} \geq 0, \\
\left.c_{S}^{2}\right|_{r_{1}=1, r_{2} \ll 1, \Omega_{r}=0} & \geq 0, & & \left.c_{S}^{2}\right|_{r_{1}=1, r_{2} \ll 1, \Omega_{r}=1} \geq 0 .
\end{array}
$$


The conditions (87)- (90) impose

$$
q>\frac{1}{2}, \quad \text { and } \quad 1 \leq p<2 q
$$

This implies that negative values of $p$ and $q$ are excluded. Furthermore, the condition (91) implies

$$
\beta>0
$$

Altogether we find the following allowed parameter space

$$
\begin{aligned}
& \left(q>\frac{1}{2}\right) \wedge(1 \leq p<2 q) \wedge \\
& \quad\left[\left(\frac{2 p-2}{6 q+3}<\alpha \leq \frac{p}{3 q} \wedge 0<\beta \leq \beta_{\max }\right) \vee\left(\frac{p}{3 q}<\alpha \leq \frac{1}{3}(4 p+8 q-2) \wedge \frac{3 \alpha q-p}{6 q}<\beta \leq \beta_{\max }\right)\right]
\end{aligned}
$$

where

$$
\beta_{\max } \equiv \frac{3 \alpha-2 p+6 \alpha q+2}{6(2 q+1)} .
$$

Although the above conditions have been derived for different initial conditions, we impose them to be true at the same time. This is because we do not know a priori initial conditions in the early Universe, as we have not specified how inflation works in these models.

To be more concrete, let us consider the theory with $p=1$ and $q=5 / 2$. In this case the dark energy equation of state along the tracker is given by $w_{\mathrm{DE}}=-1.2$ during the matter era. The condition (99) reduces to

$$
(\alpha \geq 2 \beta) \wedge\left[(\alpha>0 \wedge \beta>0 \wedge 15 \alpha \leq 2) \vee\left(15 \alpha>2 \wedge \alpha<2 \beta+\frac{2}{15} \wedge 3 \alpha \leq 22\right)\right]
$$

Adding the condition (84), the parameter space reduces to

$$
\begin{aligned}
(\alpha \geq 2 \beta) \wedge & {[(\alpha>0 \wedge 2 \sqrt{17} \sqrt{3(272-75 \alpha) \alpha+68}+561 \beta>408 \alpha+68 \wedge 75 \alpha \leq 34)} \\
& \left.\vee\left(75 \alpha>34 \wedge \alpha<2 \beta+\frac{2}{15} \wedge 3 \alpha \leq 22\right)\right]
\end{aligned}
$$

During the transition from the regime $r_{1}=1$ and $r_{2} \ll 1$ to the de Sitter attractor $\left(r_{1}=1\right.$ and $\left.r_{2}=1\right)$ the tensor propagation speed squared $c_{T}^{2}$ can be negative, depending on the model parameters. For $p=1$ and $q=5 / 2$ we have

$$
c_{T}^{2}=\frac{110+\left(22-15 \alpha-99 \beta-33 \beta \Omega_{r}\right) r_{2}+3(33 \beta-\alpha) r_{2}^{2}}{11\left[2+3(\alpha-2 \beta) r_{2}\right]\left(5+r_{2}\right)}
$$

along the tracker. Since the transition occurs at late times, the contribution of the radiation density parameter can be neglected in Eq. (103). If $|\alpha| \ll 1$ and $|\beta| \ll 1$, then $c_{T}^{2}$ is close to 1 . For positive $\alpha$ and $\beta$ of the order of unity it happens that $c_{T}^{2}$ becomes negative. Taking the derivative of Eq. (103) with respect to $r_{2}, c_{T}^{2}$ has a minimum (the second derivative $d^{2}\left(c_{T}^{2}\right) / d r_{2}^{2}$, at $r_{2}=r_{2, \min }$, is always positive in the region $0<r_{2, \text { min }}<1$ and when the conditions (101) are satisfied) at

$$
r_{2, \min }=\frac{60 \alpha-275 \beta+3 \sqrt{\Delta_{\min }}}{55 \beta-12 \alpha-594 \beta^{2}+297 \alpha \beta}
$$

where

$$
\Delta_{\min } \equiv \beta\left[180 \alpha^{2}-3 \alpha(197 \beta+8)+22 \beta(21 \beta+5)\right]
$$

In Eq. (104) we have chosen the solution with $r_{2}>0$, because another solution is always negative if the conditions (101) are satisfied.

Plugging the solution (104) into Eq. (103), we find that

$$
\left.c_{T}^{2}\right|_{r_{2, \min }}=\frac{36 \sqrt{55} \sqrt{\Delta_{\min }}-225 \alpha^{2}-15 \alpha(69 \beta+20)+66 \beta(45 \beta-23)+44}{11(30 \beta-15 \alpha+2)^{2}} .
$$




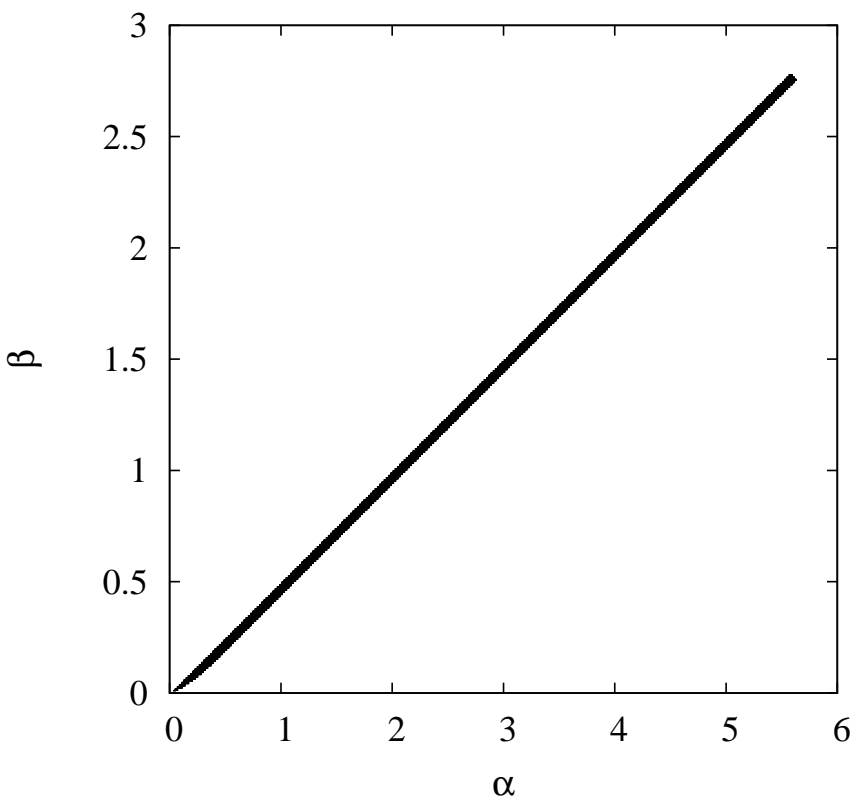

Figure 1: Allowed parameter space in the $(\alpha, \beta)$ plane (the area in black) for $p=1$ and $q=5 / 2$. We have used all the available conditions considered so far, i.e. (102) and (107).

Therefore the allowed parameter space is characterized by

$$
\Delta_{\min }<0 \vee\left[\Delta_{\min } \geq 0 \wedge\left(r_{2, \min } \leq 0 \vee r_{2, \min } \geq 1\right)\right] \vee\left[\Delta_{\min } \geq\left. 0 \wedge\left(0<r_{2, \min }<1\right) \wedge c_{T}^{2}\right|_{r_{2, \min }} \geq 0\right] .
$$

The first inequality comes from setting $r_{2, \text { min }}$ to be an imaginary number such that there is no minimum for $c_{T}^{2}$. The second inequality allows the minimum of $c_{T}^{2}$ to be outside the interested range for $r_{2}$.

In Fig. 1 w we plot the allowed parameter space for $p=1$ and $q=5 / 2$ in the $(\alpha, \beta)$ plane constrained by the conditions (102) and (107).

\section{F. Numerical simulations}

In order to confirm the analytic estimation given above, we solve the differential equations of $r_{1}, r_{2}$, and $\Omega_{r}$ numerically for given values of $\alpha, \beta, p$, and $q$. In particular we study the cosmological evolution for $p=1$ and $q=5 / 2$, in which case the parameters $\alpha$ and $\beta$ need to satisfy the constraints (102) and (107).

In Fig. 2 we plot the evolution of $\Omega_{\mathrm{DE}}, \Omega_{m}, \Omega_{r}, w_{\mathrm{eff}}$, and $w_{\mathrm{DE}}$ versus the redshift $z=1 / a-1$ for $p=1, q=5 / 2$, $\alpha=1, \beta=0.45$, and $x_{\mathrm{dS}}=1$ with the initial conditions corresponding to the tracker from the beginning $\left(r_{1}=1\right)$. For this choice of $\alpha$ and $\beta$ the conditions (102) and (107) are satisfied. Note that the coefficients $c_{i}(i=2, \cdots, 5)$ are known to be $c_{2}=9.60, c_{3}=18.07, c_{4}=4.36$, and $c_{5}=7.20$ from Eqs. (39)-(41). Furthermore both the fixed points given in Eq. (83) exist in the region $r_{a, b}>1$. Figure 2 shows that the sequence of radiation $\left(w_{\text {eff }}=1 / 3, \Omega_{r}=1\right)$, matter $\left(w_{\text {eff }}=0, \Omega_{m}=1\right)$, and de Sitter $\left(w_{\text {eff }}=-1, \Omega_{\mathrm{DE}}=1\right)$ eras is in fact realized. The dark energy density parameter grows as $\Omega_{\mathrm{DE}}=r_{2} \propto t^{2(1+s)}=t^{12 / 5}$ toward the de Sitter attractor $\left(r_{1}=r_{2}=1\right)$. As estimated by Eq. (60), the evolution of $w_{\mathrm{DE}}$ during the radiation and matter eras are given by $w_{\mathrm{DE}}=-1.267$ and $w_{\mathrm{DE}}=-1.2$, respectively. After the end of the matter-dominated epoch the solution approaches the de Sitter attractor with $w_{\mathrm{DE}}=-1$.

In Fig. 3 we show the variation of $w_{\mathrm{DE}}$ for the same model parameters as those used in Fig. 2 with a number of different initial conditions. The approach to the tracker occurs later for smaller initial values of $r_{1}$. This can be clearly seen in the numerical simulations of the cases (a)-(c) in Fig. 3. The case (a) corresponds to the early tracking, whereas the case (c) to the late-time tracking with smaller initial values of $r_{1}$. In the regime characterized by $r_{1} \ll 1$ and $r_{2} \ll 1$, the analytic estimation (78) gives $w_{\mathrm{DE}}=-0.0625$ and $w_{\mathrm{DE}}=-0.03125$ during the radiation and the matter eras, respectively. These analytic values of $w_{\mathrm{DE}}$ are in good agreement with their numerical values for the late-time tracking solution (such as the case (c) in Fig. 3).

In Fig. 4 the evolution of the quantity $Q_{S}$ is shown for the same model parameters and the initial conditions as those given in Fig. 3. For the tracker our numerical simulations show that $Q_{S}$ grows according to Eq. (62) in the 


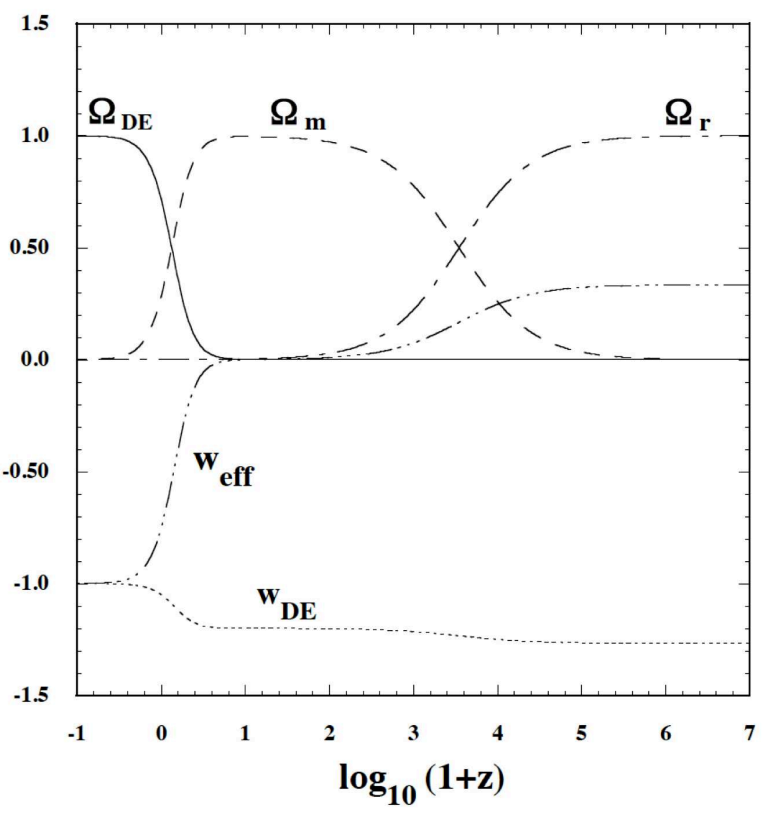

Figure 2: Evolution of the density parameters $\Omega_{\mathrm{DE}}, \Omega_{m}, \Omega_{r}, w_{\mathrm{eff}}$, and $w_{\mathrm{DE}}$ versus the redshift $z$ for $p=1, q=5 / 2, \alpha=1$, $\beta=0.45$, and $x_{\mathrm{dS}}=1$. The initial conditions are chosen to be $r_{1}=1, r_{2}=10^{-30}$, and $\Omega_{r}=0.9998$ at $z=1.76 \times 10^{7}$. In this case the solution is on the tracker from the beginning.

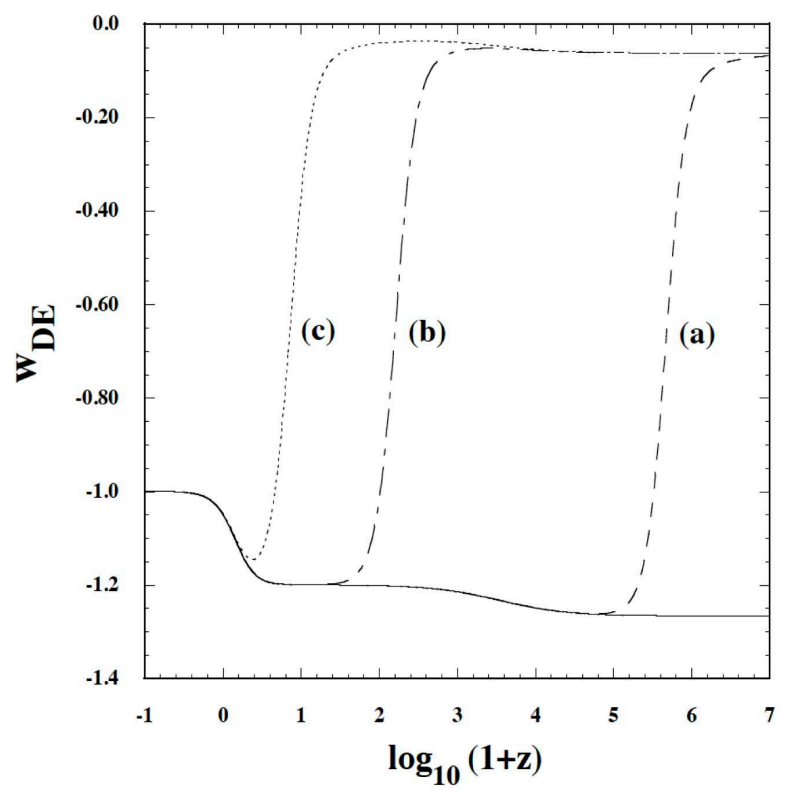

Figure 3: Variation of $w_{\mathrm{DE}}$ versus $z$ for $p=1, q=5 / 2, \alpha=1, \beta=0.45$, and $x_{\mathrm{dS}}=1$ with several different initial conditions. The solid line corresponds to the tracker with the initial conditions same as those given in Fig. 2 The initial conditions for the cases (a)-(c) are (a) $r_{1}=4.0 \times 10^{-2}, r_{2}=5.0 \times 10^{-26}, \Omega_{r}=0.9998$ at $z=1.82 \times 10^{7}$, (b) $r_{1}=1.0 \times 10^{-5}, r_{2}=1.0 \times 10^{-13}$, $\Omega_{r}=0.9998$ at $z=1.76 \times 10^{7}$, and (c) $r_{1}=1.0 \times 10^{-7}, r_{2}=1.0 \times 10^{-9}, \Omega_{r}=0.99995$ at $z=6.64 \times 10^{7}$, respectively.

regime $r_{2} \ll 1$ (i.e. $Q_{S} \propto r_{2} \propto t^{12 / 5}$ ), which finally approaches the value (68) at the de Sitter solution. For the initial conditions with $r_{1} \ll 1$ and $r_{2} \ll 1$ we find that the early evolution of $Q_{S}$ is well described by Eq. (79), i.e. $Q_{S} \propto r_{2} \propto t^{19 / 32}$ during the radiation era. As we can see in Fig. 团 the evolution of $Q_{S}$ shifts to that of the tracker after the solutions reach the regime around $r_{1}=1$. Provided that $r_{2}>0$ initially, $Q_{S}$ always remains to be positive.

As we see in Eqs. (64) and (81) the quantity $Q_{T} / M_{\mathrm{pl}}^{2}$ is close to the value $1 / 4$ in the regime $r_{2} \ll 1$, independently 


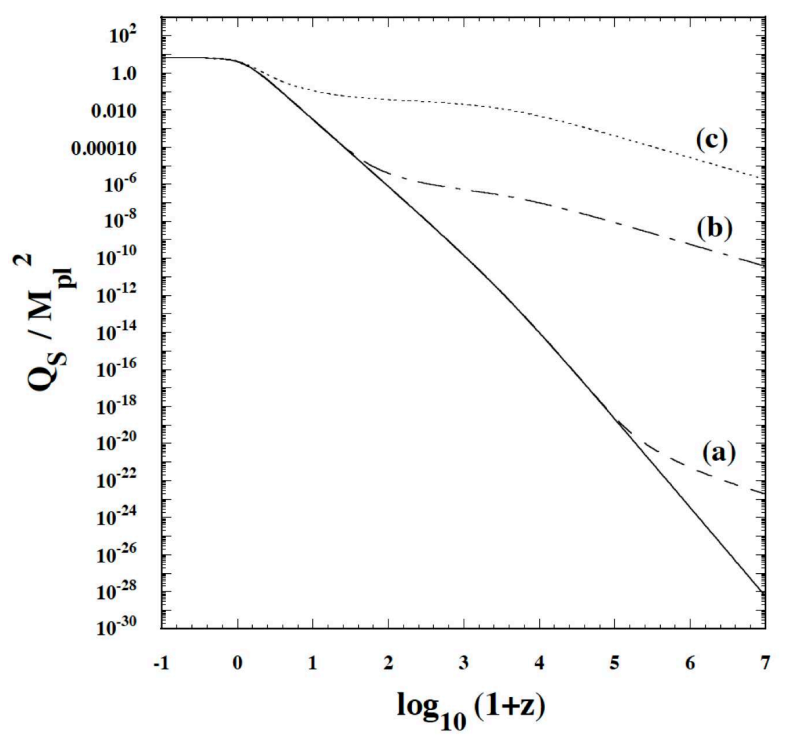

Figure 4: Variation of $Q_{S} / M_{\mathrm{pl}}^{2}$ versus $z$ for the same model parameters and initial conditions as those given in Fig. 3 The solid line represents the tracker solution, whereas the cases (a), (b), and (c) correspond to the evolution for the initial conditions as those given in Fig. 3

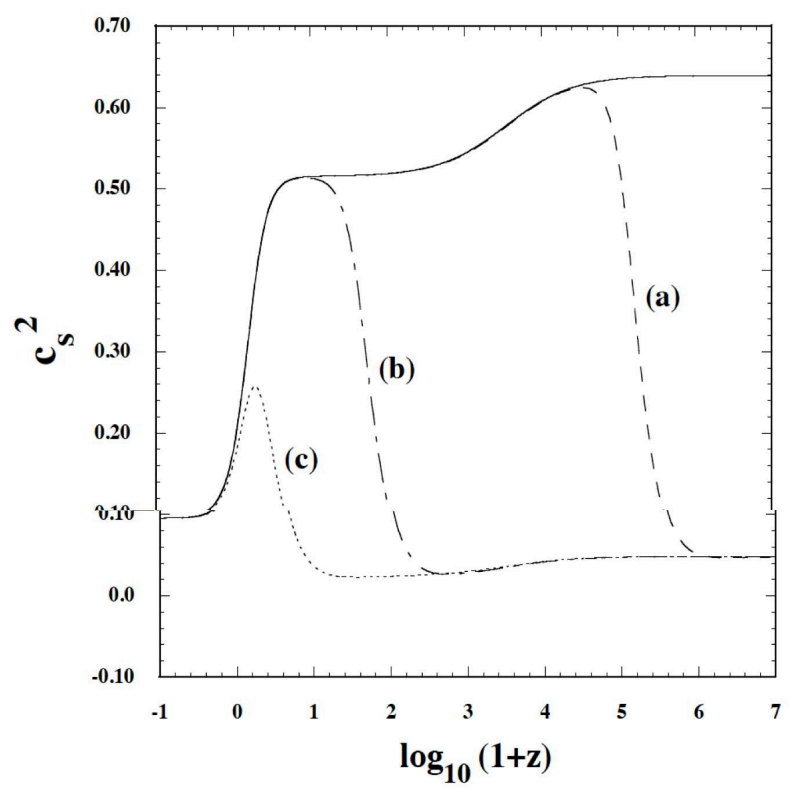

Figure 5: Evolution of $c_{S}^{2}$ versus $z$ for the same model parameters and initial conditions as those given in Fig. 3 The solid line represents the tracker solution, whereas the cases (a), (b), and (c) correspond to the evolution for the initial conditions as those given in Fig. 3

of the values of $r_{1}$. Numerically we confirmed that, for both the initial conditions $r_{1}=1$ and $r_{1} \ll 1, Q_{T} / M_{\mathrm{pl}}^{2}$ stays the value around $1 / 4$ until recently and then it finally approaches the value (70) at the de Sitter solution. Provided that $3 \alpha-6 \beta+2>0$ the no-ghost condition for the tensor perturbation is always satisfied.

In Fig. [5 we illustrate the evolution of $c_{S}^{2}$ for the same model parameters and the initial conditions as those given in Fig. 3. The analytic estimation (63) for the tracker gives $c_{S}^{2}=0.639$ and $c_{S}^{2}=0.515$ during the radiation and matter dominated epochs respectively, which show good agreement with the numerical result in Fig. [5 Finally $c_{S}^{2}$ approaches the value $9.48 \times 10^{-2}$ with a smooth transition from the matter era to the de Sitter epoch. In the regime $r_{1} \ll 1$ and $r_{2} \ll 1$ the analytic estimation (80) gives $c_{S}^{2} \simeq(13 / 544)\left(1+\Omega_{r}\right)$. In fact the numerical simulations for the cases (a), 


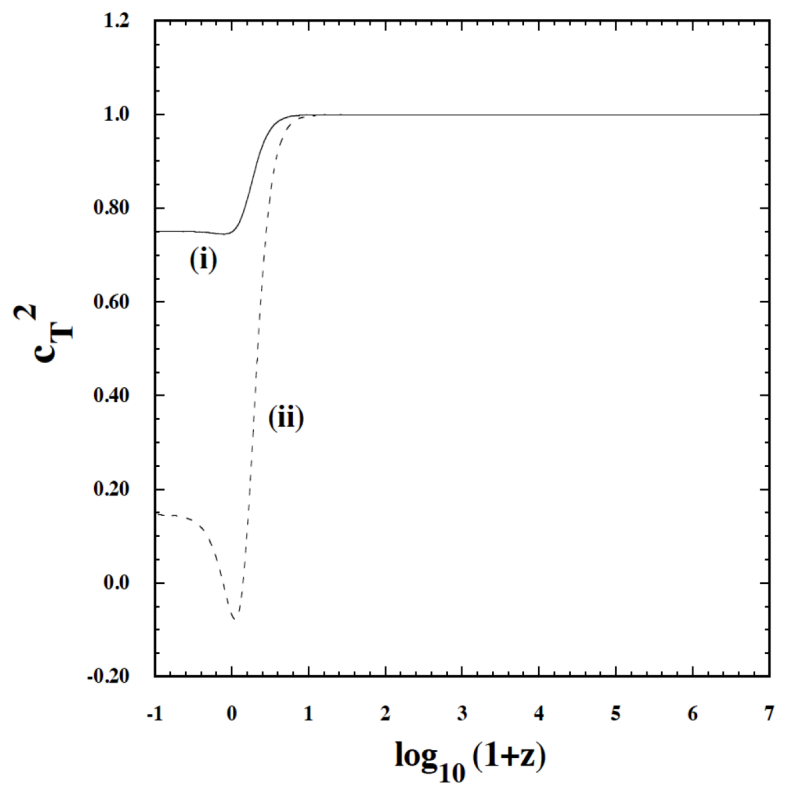

Figure 6: Evolution of $c_{T}^{2}$ versus $z$ along the tracker for (i) $p=1, q=5 / 2, \alpha=1, \beta=0.45, x_{\mathrm{dS}}=1$, and (ii) $p=1, q=5 / 2$, $\alpha=6.1, \beta=3, x_{\mathrm{dS}}=1$. In both cases the initial conditions are chosen to be $r_{1}=1, r_{2}=10^{-30}$, and $\Omega_{r}=0.9998$. In the case (ii) the condition (107) is violated, so that $c_{T}^{2}$ temporally becomes negative during the transition from the regime $r_{2} \ll 1$ to the regime $r_{2}=1$.

(b), and (c) reproduce this value before the solutions reach the tracker. Since $0<c_{S}^{2}<1$ from the radiation era to the de Sitter epoch, the Laplacian instability of the scalar perturbation is absent.

Figure [6] depicts the evolution of $c_{T}^{2}$ for the tracker with two different combinations of $\alpha$ and $\beta$. The case (i) corresponds to the model parameters and the initial conditions as those given in Fig. 2. As estimated by Eq. (65), the tensor propagation speed squared along the tracker is very close to 1 but slightly less than 1 in the early cosmological epoch. In the case (i) the model parameters satisfy the condition (107), so that $c_{T}^{2}$ takes a positive minimum value 0.745 at $r_{2}=0.847$. Then the solution finally approaches the de Sitter attractor with $c_{T}^{2}=0.751$. In the case (ii), in which the condition (107) is violated, the minimum value of $c_{T}^{2}$ is negative. This shows that the condition (107) is in fact required to avoid the Laplacian instability of the tensor perturbation. We have also run our numerical code for the initial conditions with $r_{1} \ll 1, r_{2} \ll 1$ and found that the evolution of $c_{T}^{2}$ is similar to that for the tracker. The only difference is that the tensor propagation speed is slightly superluminal in the regime $r_{1} \ll 1, r_{2} \ll 1$. In fact, Eq. (82) shows that $c_{T}^{2}$ is slightly larger than 1 under the conditions (97) and (98).

We have also carried out the numerical simulations for other values of $p, q$ and confirmed the accuracy of the analytic estimation. If we choose larger values of $q$ for given $p$, the dark energy equation of state $w_{\mathrm{DE}}$ along the tracker approaches -1 . In the limit where $q \rightarrow \infty$ the scalar propagation speed squared (69) at the de Sitter solution reduces to $c_{S}^{2} \rightarrow \alpha-2 \beta$ and hence we require the condition $\alpha \geq 2 \beta$. On the other hand the condition (94) reduces to $\alpha<2 \beta$ for $q \rightarrow \infty$. Hence, if $w_{\mathrm{DE}}$ along the tracker is close to -1 , the allowed parameter space tends to be smaller.

\section{CONCLUSIONS}

In the Horndeski's most general scalar-tensor theories we derived conditions for the avoidance of ghosts and Laplacian instabilities associated with scalar, tensor, and vector perturbations. The four conditions (23), (25), (27), and (28) need to be satisfied for the theoretical consistency. Vector perturbations do not give rise to any additional conditions to those derived for scalar and tensor perturbations.

The Horndeski's action covers most of the dark energy models proposed in literature and hence our formulas are general enough to apply them to concrete models with second-order field equations. We proposed new kinetically driven dark energy models described by the functions (30), which cover the covariant Galileon as a specific case. For the choice of the powers $p_{i}(i=2,3,4,5)$ given in Eq. (36) we showed the existence of the tracker solution along which $H \dot{\phi}^{2 q}=$ constant. Finally the solutions approach a stable de Sitter attractor at which $\dot{\phi}=$ constant.

Along the tracker the dark energy equation of state during the matter dominance is found to be $w_{\mathrm{DE}}=-1-p /(2 q)$. 
The covariant Galileon $(p=1$ and $q=1 / 2)$ corresponds to $w_{\mathrm{DE}}=-2$ during the matter era, which is not favored from the combined data analysis of SNIa, CMB, and BAO. The extended Galileon model we proposed can alleviate this problem because $w_{\mathrm{DE}}$ can be close to -1 for $p$ smaller than $q$.

We clarified the theoretically allowed parameter space in which the ghosts and Laplacian instabilities are absent. For $p=1$ and $q=5 / 2$ we carried out numerical simulations to check the evolution of the background quantities (like $w_{\mathrm{DE}}$ and $\Omega_{\mathrm{DE}}$ ) as well as the quantities such as $c_{S}^{2}, c_{T}^{2}, Q_{S}$, and $Q_{T}$. As we estimated analytically, the dark energy equation of state for the tracker evolves as $w_{\mathrm{DE}}=-1.267$ (radiation era), $w_{\mathrm{DE}}=-1.2$ (matter era), and $w_{\mathrm{DE}}=-1$ (de Sitter era), see Fig. 3. For the initial conditions with $r_{1} \ll 1, w_{\mathrm{DE}}$ starts to evolve from the value estimated by Eq. (78). The approach to the tracker occurs later for smaller initial values of $r_{1}$.

For the values of $\alpha$ and $\beta$ which are inside the allowed parameter space, our numerical simulations show that $c_{S}^{2}$, $c_{T}^{2}, Q_{S}$, and $Q_{T}$ remain to be positive in the cosmic expansion history. Note that the condition (107) is important to avoid that $c_{T}^{2}$ becomes negative during the transition from the matter era to the de Sitter epoch. While we showed the cosmological evolution for one choice of $p$ and $q$, we also confirmed that the analytic estimation is trustable for other values of $p$ and $q$. In the limit that $p / q \rightarrow 0$ the dark energy equation of state for the tracker mimics that in the $\Lambda$ CDM model.

It will be of interest to see how the combined data analysis of SNIa, CMB, and BAO places constraints on the tracker solution in the extended Galileon models. In order to confront this model with the observations of large scale structure and weak lensing, we also need to study the evolution of matter density perturbations as well as gravitational potentials. We leave these issues for future work.

\section{ACKNOWLEDGEMENTS}

A.D.F. and S. T. were supported by the Grant-in-Aid for Scientific Research Fund of the JSPS Nos. 10271 and 30318802. A. D. F. is also supported by the ThEP Center. S. T. also thanks financial support for the Grant-in-Aid for Scientific Research on Innovative Areas (No. 21111006). We thank Alexander Vikman for his initial collaboration on this work. S. T. is grateful to the organizers of sixth Aegean summer school and the Dark Universe Conference for their kind hospitalities during which a part of this work was done.

\section{Appendix A: Ghost conditions for the vector modes}

The no-ghost conditions for the vector modes in the presence of two perfect fluids can be found by using the method in Ref. [35]. Let us consider the perturbed metric

$$
d s^{2}=-d t^{2}+a \gamma_{i} d t d x^{i}+a^{2}\left(\delta_{i j}+C_{i, j}+C_{j, i}\right) d x^{i} d x^{j},
$$

where $\gamma_{i, i}=0=C_{i, i}$. In order to describe the vector perturbations at linear order, for the perfect fluid we write $\mu u_{\alpha}^{A}=\mathcal{A}^{A} \partial_{\alpha} \mathcal{B}_{A}$, where $\mathcal{A}^{A}$ and $\mathcal{B}_{A}$ are the velocity potentials for the fluid $A$. We can choose the background values for $\mathcal{A}^{A}$ and $\mathcal{B}_{A}$ as follows: $\mathcal{A}^{A}=0$, and $\partial_{i} \mathcal{B}_{A}=b_{i}^{A}=$ arbitrary constant. Then, for a plane wave in Fourier space, we have $C_{i} k_{i}=0$. By splitting $\mathcal{B}^{B}$ as $\mathcal{B}^{B}=b_{i}^{B} x^{i}+b_{i}^{B} \delta \mathcal{B}_{B}$, and choosing the gauge for which $C_{\perp}=0=\delta \mathcal{B}_{A}$, (where $C_{\perp}$ is the component of $C_{i}$ perpendicular to $b_{i}^{B}$ ), and the arbitrary background quantities such that $b_{i}^{B} k_{i}=0$, we find that $b_{i}^{B}$ is parallel to $C_{i}$ and both are perpendicular to $k_{i}$.

After expanding the action at second order in the fields and integrating out the auxiliary fields, we obtain

$$
S=\int d^{4} x\left[Q_{11}^{V} \dot{C}_{i} \dot{C}_{i}+2 Q_{12}^{V} b_{i}^{B} \dot{C}_{i} \dot{\delta} \dot{\mathcal{B}}_{B}+Q_{22}^{V} b_{i}^{B} b_{i}^{B} \dot{\delta}_{B}^{2}\right]
$$

where $Q_{11}, Q_{12}$, and $Q_{22}$ are time-dependent coefficients. The two no-ghost conditions can be written as

$$
\begin{aligned}
Q_{11}^{V} & =a^{5} \frac{w_{1}(k / a)^{2}\left[\left(1+w_{A}\right) \rho_{A}+\rho_{B}\left(1+w_{B}\right)\right]}{2\left[2\left(1+w_{A}\right) \rho_{A}+2 \rho_{B}\left(1+w_{B}\right)+w_{1}(k / a)^{2}\right]}>0, \\
Q_{11}^{V} Q_{22}^{V}-\left(Q_{12}^{V}\right)^{2} & =a^{10} \frac{w_{1}(k / a)^{2}\left(1+w_{A}\right) \rho_{A}\left(1+w_{B}\right) \rho_{B}}{4\left[2\left(1+w_{A}\right) \rho_{A}+2\left(1+w_{B}\right) \rho_{B}+w_{1}(k / a)^{2}\right]}>0 .
\end{aligned}
$$

These conditions are satisfied for $w_{1}>0$ (which corresponds to the condition (27)) and $\left(1+w_{A}\right) \rho_{A}>0$, and $\left(1+w_{B}\right) \rho_{B}>0$. In General Relativity with one single perfect fluid, only the condition (A3) holds, and it agrees with 
the result in Ref. [35], when $w_{1}=M_{\mathrm{pl}}^{2}$. Hence the vector perturbations do not provide additional constraints to those derived for scalar and tensor perturbations.

[1] S. Weinberg, Rev. Mod. Phys. 61, 1-23 (1989).

[2] V. Sahni and A. A. Starobinsky, Int. J. Mod. Phys. D 9, 373 (2000); S. M. Carroll, Living Rev. Rel. 4, 1 (2001); T. Padmanabhan, Phys. Rept. 380, 235 (2003); P. J. E. Peebles and B. Ratra, Rev. Mod. Phys. 75, 559 (2003); E. J. Copeland, M. Sami and S. Tsujikawa, Int. J. Mod. Phys. D 15, 1753 (2006); S. Tsujikawa, arXiv:1004.1493 [astro-ph.CO].

[3] Y. Fujii, Phys. Rev. D 26, 2580 (1982); L. H. Ford, Phys. Rev. D 35, 2339 (1987); C. Wetterich, Nucl. Phys B. 302, 668 (1988); B. Ratra, P. J. E. Peebles, Phys. Rev. D37, 3406 (1988); T. Chiba, N. Sugiyama and T. Nakamura, Mon. Not. Roy. Astron. Soc. 289, L5-L9 (1997); R. R. Caldwell, R. Dave and P. J. Steinhardt, Phys. Rev. Lett. 80, 1582 (1998).

[4] C. Armendariz-Picon, T. Damour and V. F. Mukhanov, Phys. Lett. B458, 209-218 (1999); T. Chiba, T. Okabe and M. Yamaguchi, Phys. Rev. D62, 023511 (2000); C. Armendariz-Picon, V. F. Mukhanov and P. J. Steinhardt, Phys. Rev. Lett. 85, 4438-4441 (2000).

[5] S. Capozziello, Int. J. Mod. Phys. D 11, 483 (2002); S. Capozziello, S. Carloni and A. Troisi, Recent Res. Dev. Astron. Astrophys. 1, 625 (2003); S. M. Carroll, V. Duvvuri, M. Trodden and M. S. Turner, Phys. Rev. D 70, 043528 (2004).

[6] W. Hu and I. Sawicki, Phys. Rev. D 76, 064004 (2007); A. A. Starobinsky, JETP Lett. 86, 157 (2007); S. A. Appleby and R. A. Battye, Phys. Lett. B 654, 7 (2007); L. Amendola and S. Tsujikawa, Phys. Lett. B 660, 125 (2008); S. Tsujikawa, Phys. Rev. D 77, 023507 (2008).

[7] S. M. Carroll, A. De Felice, V. Duvvuri, D. A. Easson, M. Trodden and M. S. Turner, Phys. Rev. D71, 063513 (2005); S. Nojiri, S. D. Odintsov, Phys. Lett. B631, 1-6 (2005); I. Navarro and K. Van Acoleyen, JCAP 0603, 008 (2006); A. De Felice and S. Tsujikawa, Phys. Lett. B675, 1-8 (2009); A. De Felice and T. Suyama, JCAP 0906, 034 (2009); A. De Felice and T. Suyama, Phys. Rev. D80, 083523 (2009); A. De Felice, D. F. Mota and S. Tsujikawa, Phys. Rev. D81, 023532 (2010).

[8] C. Brans and R. H. Dicke, Phys. Rev. 124, 925 (1961).

[9] G. R. Dvali, G. Gabadadze and M. Porrati, Phys. Lett. B 485, 208 (2000).

[10] A. Nicolis, R. Rattazzi and E. Trincherini, Phys. Rev. D79, 064036 (2009).

[11] C. Deffayet, G. Esposito-Farese and A. Vikman, Phys. Rev. D79, 084003 (2009); C. Deffayet, S. Deser and G. EspositoFarese, Phys. Rev. D 80, 064015 (2009).

[12] T. P. Sotiriou and V. Faraoni, Rev. Mod. Phys. 82, 451 (2010); A. De Felice and S. Tsujikawa, Living Rev. Rel. 13, 3 (2010); S. Tsujikawa, Lect. Notes Phys. 800, 99-145 (2010); T. Clifton, P. G. Ferreira, A. Padilla and C. Skordis, arXiv:1106.2476 [astro-ph.CO].

[13] J. O'Hanlon, Phys. Rev. Lett. 29, 137 (1972); T. Chiba, Phys. Lett. B575, 1-3 (2003).

[14] A. De Felice and T. Tanaka, Prog. Theor. Phys. 124, 503-515 (2010); A. De Felice, T. Suyama and T. Tanaka, Phys. Rev. D83, 104035 (2011).

[15] C. Deffayet, G. R. Dvali, G. Gabadadze and A. I. Vainshtein, Phys. Rev. D 65, 044026 (2002); M. Porrati, Phys. Lett. B 534, 209 (2002); M. A. Luty, M. Porrati and R. Rattazzi, JHEP 0309, 029 (2003).

[16] A. De Felice and S. Tsujikawa, Phys. Rev. Lett. 105, 111301 (2010).

[17] A. De Felice and S. Tsujikawa, arXiv:1008.4236 [hep-th] (Physical Review D to appear).

[18] A. I. Vainshtein, Phys. Lett. B 39, 393 (1972).

[19] C. Deffayet, X. Gao, D. A. Steer and G. Zahariade, arXiv:1103.3260 [hep-th].

[20] G. W. Horndeski, Int. J. Theor. Phys. 10, 363-384 (1974).

[21] T. Kobayashi, M. Yamaguchi and J. Yokoyama, arXiv:1105.5723 [hep-th].

[22] C. Charmousis, E. J. Copeland, A. Padilla and P. M. Saffin, arXiv:1106.2000 [hep-th].

[23] S. M. Carroll et al., Phys. Rev. D 71, 063513 (2005); S. Nojiri, S. D. Odintsov and M. Sasaki, Phys. Rev. D 71, 123509 (2005); G. Calcagni, S. Tsujikawa and M. Sami, Class. Quant. Grav. 22, 3977 (2005); A. De Felice, M. Hindmarsh and M. Trodden, JCAP 0608, 005 (2006).

[24] N. Chow and J. Khoury, Phys. Rev. D80, 024037 (2009); F. P. Silva and K. Koyama, Phys. Rev. D 80, 121301 (2009); C. de Rham and A. J. Tolley, JCAP 1005, 015 (2010); T. Kobayashi, H. Tashiro and D. Suzuki, Phys. Rev. D 81, 063513 (2010); T. Kobayashi, Phys. Rev. D81, 103533 (2010); A. De Felice and S. Tsujikawa, JCAP 1007, 024 (2010); A. De Felice, S. Mukohyama and S. Tsujikawa, Phys. Rev. D82, 023524 (2010); K. Hinterbichler, M. Trodden and D. Wesley, Phys. Rev. D82, 124018 (2010); T. Kobayashi, M. Yamaguchi and J. Yokoyama, Phys. Rev. Lett. 105, 231302 (2010); D. F. Mota, M. Sandstad and T. Zlosnik, JHEP 1012, 051 (2010); C. Burrage, C. de Rham, D. Seery and A. J. Tolley, JCAP 1101, 014 (2011); K. Hirano and Z. Komiya, arXiv:1012.5451 [astro-ph.CO]; G. Goon, K. Hinterbichler and M. Trodden, arXiv:1109.3450 [hep-th]; Z. -G. Liu, J. Zhang and Y. S. Piao, arXiv:1105.5713 [astro-ph.CO]; H. Wang, T. Qiu and Y. S. Piao, arXiv:1110.1795 [hep-ph].

[25] C. Deffayet, O. Pujolas, I. Sawicki and A. Vikman, JCAP 1010, 026 (2010); O. Pujolas, I. Sawicki, A. Vikman, arXiv:1103.5360 [hep-th].

[26] R. Kimura and K. Yamamoto, JCAP 1104, 025 (2011).

[27] R. Kimura, T. Kobayashi and K. Yamamoto, arXiv:1110.3598 [astro-ph.CO].

[28] L. Amendola, Phys. Lett. B 301, 175 (1993); C. Germani and A. Kehagias, Phys. Rev. Lett. 105, 011302 (2010); Phys. 
Rev. Lett. 106, 161302 (2011); C. Germani and Y. Watanabe, JCAP 1107, 031 (2011); G. Gubitosi, E. V. Linder, Phys. Lett. B703, 113-118 (2011).

[29] A. De Felice, T. Kobayashi and S. Tsujikawa, arXiv:1108.4242 [gr-qc].

[30] X. Gao and D. A. Steer, arXiv:1107.2642 [astro-ph.CO].

[31] A. De Felice and S. Tsujikawa, Phys. Rev. D 84, 083504 (2011).

[32] X. Gao, T. Kobayashi, M. Yamaguchi and J. Yokoyama, arXiv:1108.3513 [astro-ph.CO].

[33] M. Ostrogradski: Mem. Ac. St. Petersbourg VI 4, 385 (1850).

[34] J. M. Bardeen, Phys. Rev. D 22, 1882 (1980).

[35] A. De Felice, J. M. Gerard and T. Suyama, Phys. Rev. D81, 063527 (2010); A. De Felice, J. M. Gerard and T. Suyama, Phys. Rev. D82, 063526 (2010).

[36] A. De Felice, R. Kase and S. Tsujikawa, Phys. Rev. D 83, 043515 (2011).

[37] S. Nesseris, A. De Felice and S. Tsujikawa, Phys. Rev. D 82, 124054 (2010).

[38] G. Dvali and M. S. Turner, astro-ph/0301510 